\title{
Knockdown of Son, a mouse homologue of the ZTTK syndrome gene, causes neuronal migration defects and dendritic spine abnormalities
}

Masashi Ueda', Tohru Matsuki ${ }^{1}$, Masahide Fukada', Shima Eda ${ }^{1}$, Akie Toya ${ }^{1,2}$, Akio lio $^{1,3}$, Hidenori Tabata ${ }^{4}$ and Atsuo Nakayama ${ }^{1,2^{*}}$ (D)

\begin{abstract}
Zhu-Tokita-Takenouchi-Kim (ZTTK) syndrome, a rare congenital anomaly syndrome characterized by intellectual disability, brain malformation, facial dysmorphism, musculoskeletal abnormalities, and some visceral malformations is caused by de novo heterozygous mutations of the SON gene. The nuclear protein SON is involved in gene transcription and RNA splicing; however, the roles of SON in neural development remain undetermined. We investigated the effects of Son knockdown on neural development in mice and found that Son knockdown in neural progenitors resulted in defective migration during corticogenesis and reduced spine density on mature cortical neurons. The induction of human wild-type SON expression rescued these neural abnormalities, confirming that the abnormalities were caused by SON insufficiency. We also applied truncated SON proteins encoded by disease-associated mutant SON genes for rescue experiments and found that a truncated SON protein encoded by the most prevalent SON mutant found in ZTTK syndrome rescued the neural abnormalities while another much shorter mutant SON protein did not. These data indicate that SON insufficiency causes neuronal migration defects and dendritic spine abnormalities, which seem neuropathological bases of the neural symptoms of ZTTK syndrome. In addition, the results support that the neural abnormalities in ZTTK syndrome are caused by SON haploinsufficiency independent of the types of mutation that results in functional or dysfunctional proteins.
\end{abstract}

Keywords: SON, Zhu-Tokita-Takenouchi-Kim syndrome, Brain malformation, Intellectual disability, Neuronal migration, Spinogenesis

\section{Introduction}

Recent genetic studies identified 31 individuals exhibiting intellectual disability (ID) and/or developmental delay with de novo heterozygous mutations in $S O N$, which was established as Zhu-Tokita-Takenouchi-Kim (ZTTK) syndrome

\footnotetext{
* Correspondence: atsuon@inst-hsc.jp

'Department of Cellular Pathology, Institute for Developmental Research, Aichi Developmental Disability Center, Kasugai, Aichi 4800392, Japan

${ }^{2}$ Department of Neurobiochemistry, Nagoya University School of Medicine, Nagoya, Aichi 4668560, Japan

Full list of author information is available at the end of the article
}

[1-6]. ZTTK syndrome was further characterized as a congenital anomaly syndrome of ID, brain malformation, facial dysmorphism, musculoskeletal abnormalities, and less common visceral malformations $[1,2,4]$. The mutations found to be associated with ZTTK syndrome are mostly frameshift mutations and nonsense substitutions generating a premature termination codon [1-6], and transcripts of the mutant gene seem to be degraded due to nonsensemediated mRNA decay (NMD) [1]; this has made ZTTK syndrome to be regarded as an entity caused by $S O N$ haploinsufficiency. 
SON is a ubiquitously expressed and evolutionarily conserved gene in vertebrates and is located on the human chromosome region 21q22.11 [4]. It encodes the DNA- and RNA-binding protein SON, which functions in RNA splicing as well as gene repression [7-13]. A wide variety of genes are, thus, under the control of SON, and SON has been reported to be involved in cell cycle regulation and stem cell maintenance [7-12]. However, the functional significance of SON in neural development is largely unknown, and the pathological consequence of SON haploinsufficiency underlying the neural phenotypes of ZTTK syndrome, such as ID and brain malformation, remains undetermined. In this report, we revealed through knockdown experiments in the developing mouse brain that Son insufficiency caused neuronal migration abnormalities and reduced spine density. Rescue experiments that induced the expression of human wild-type SON protein and truncated SON proteins encoded by disease-associated mutant $S O N$ genes provided further information relevant to the pathophysiology of ZTTK syndrome.

\section{Materials and methods}

\section{Animals}

All animals were used in accordance with an animal protocol approved by the Animal Care and Use Committee of the Institute for Developmental Research, Aichi Developmental Disability Center. Timed-pregnant ICR mice were purchased from Japan SLC (Hamamatsu, Japan).

\section{Antibodies}

We raised an antibody against mouse $\mathrm{SON}$ by immunizing rabbits with a keyhole limpet hemocyanin-fused mouse SON peptide (NM_178880, residues 4-23) (Biomatik, Wilmington, DE). The other antibodies used in this study were as follows: anti-HA $(6 \mathrm{E} 2,2367)$ (Cell Signaling Technology, Beverly, MA), anti- $\beta$-actin (AC-15, A5441), anti-SRSF2/SC35 (SC-35, SAB4200725) (SigmaAldrich, St. Louis, MO), anti-green fluorescent protein (GFP) (A10262), Alexa-conjugated secondary antibodies (Invitrogen, Waltham, MA), and horseradish peroxidase (HRP)-conjugated secondary antibodies (Jackson ImmunoResearch, West Grove, PA).

\section{Plasmid construction}

Short hairpin RNA (shRNA) vectors were constructed by inserting cDNAs of the following shRNAs into the pLLC vector [14]: shRNA\#1 (5'-AGGCTCAATTACTT GAAATA-3') [8] and shRNA\#2 (5'-GCTGAGCGTT CTATGATGT-3') [15]. The pCAGGS vector, kindly provided by Dr. Miyazaki in Osaka University, was engineered to express HA-tagged human SON (hSON), shRNA-resistant human SON (hSONr), or a disease- associated mutant SON (hSONm1 or hSONm2). hSONr was derived from $S O N$ cDNA containing three nucleotide substitutions within the target sequence of shRNA\#1.

\section{Cell culture}

Neuro-2a and HEK293 cells were obtained from ATCC (Manassas, VA) and RIKEN BRC (Tsukuba, Japan), respectively. Each cell line was maintained with DMEM supplemented with $10 \% \mathrm{FBS}$, penicillin, and streptomycin under standard conditions. The expression plasmids were transfected with polyethyleneimine (Polysciences, Inc., Warrington, PA) or Lipofectamine 2000 (Invitrogen), according to manufacturer's directions.

\section{In utero electroporation (IUE)}

Various combinations of plasmids were transfected into neural progenitors on the lateral ventricular surface of E14.5 embryos by IUE as previously described [16]. Electroporation was performed by administering five consequent electronic pulses at an intensity of $35 \mathrm{~V}$ for a duration of $50 \mathrm{~ms}$ with 450-ms intervals using a NEPA21 SuperElectroporator (NEPA Gene, Chiba, Japan). For neuronal migration analysis, $1 \mu \mathrm{g}$ of shRNA vector with $1.5 \mu \mathrm{g}$ of the pCAGGS vectors harboring the various forms of human SON cDNA described above were applied. For dendritic spine formation analysis, a plasmid mixture containing $1 \mu \mathrm{g}$ of shRNA vector with or without $0.5 \mu \mathrm{g}$ of the hSON expression vectors was applied.

\section{Immunocytochemistry and immunohistochemistry}

Neuro-2a cells were grown on poly-L-lysine coated glass coverslips. The cells were fixed with $4 \%$ paraformaldehyde and permeabilized with $0.1 \%$ Triton X-100. Immunocytochemical staining was performed with the anti-SON antibody and Alexa-conjugated secondary antibodies (Invitrogen).

Paraffin-embedded brain tissues were sectioned at a thickness of $4 \mu \mathrm{m}$ and subjected to immunohistochemical staining and hematoxylin and eosin (HE) staining. Anti-SON immunoreactivity was visualized using EnVision (Dako, Glostrup, Denmark). All images were acquired with BX60 microscope (Olympus, Tokyo, Japan).

\section{Neuronal migration analysis and spine density analysis} Mice subjected to IUE were sacrificed at E18.5 or P60, and perfused with $4 \%$ paraformaldehyde in PBS. Then the mice were dissected and obtained brains were postfixed in paraformaldehyde solution for $2-24 \mathrm{~h}$. The brains were embedded in 3\% agarose and sectioned at a thickness of $50-100 \mu \mathrm{m}$ using a VT1200S vibrating microtome (Leica Microsystems Wetzlar, Germany). The coronal sections were stained with an anti-GFP antibody to visualize Son knockdown cells. The distribution of GFP-positive cells at E18.5 was examined to 
assess neuronal migration. For spine density analysis, dendrites of pyramidal neurons in cortical layer II/III at P60 were examined. The number of spines on each dendrite at between $30 \mu \mathrm{m}$ and $80 \mu \mathrm{m}$ from the soma was counted, and the spine density was represented as the number of spines per dendrite length of $10 \mu \mathrm{m}$. All images were acquired using a FV1000 (Olympus) or LSM880 (Carl Zeiss, Göttingen, Germany) confocal laser scanning microscope. Image processing was performed with Fiji (http://fiji.sc) and Photoshop (Adobe Systems, San Jose, CA).

\section{Statistical analyses}

Statistical significance was determined using one-way ANOVA followed by a Dunnett's post hoc test for multiple comparisons using Prism 8 (GraphPad Software, San Diego, CA).

\section{Results}

The human SON and mouse Son genes encode 2426amino-acid and 2444-amino-acid proteins, respectively, and share $84.2 \%$ homology [13] (Fig. 1a). We raised an antibody against mouse SON and found that it recognized a major band of approximately $260 \mathrm{kDa}$, which corresponds to the predicted size, and a few lower molecular weight bands upon Western blot analysis of mouse embryonic brain lysates (Fig. 1b). These bands were almost completely absent after the antibody was preabsorbed with the antigen peptide, confirming its specificity (Fig. 1b, right panel). In immunocytochemistry, the antibody recognized nuclear speckles in Neuro-2a cells, and the signals were partially colocalized with SRSF2, a splicing factor that, with SON, forms the core of speckles [17], further confirming the specificity of the antibody (Fig. 1c). The antibody worked well for immunohistochemistry as well, and we found that the most cells in the developing mouse brain at E15.5 expressed SON and that SON signals were especially conspicuous as nuclear speckles in presumptive migrating neuronal progenitors in the intermediate zone (IZ) and neurons in the cortical plate (Fig. 1d). In addition, we confirmed that the SON expression was maintained in mature neurons at P60 (Fig. 1d).

To reveal the functional significance of SON in neural progenitors, we then applied IUE to knockdown Son specifically in these cells and examined the effects on migration. We generated two shRNA expression constructs targeting independent sites of Son mRNA (Fig. 2a) and confirmed that both shRNAs reduced SON expression levels in Neuro-2a cells to $17.8 \%$ (shRNA\#1) and $32.6 \%$ (shRNA\#2) of that in the control (Fig. 2b). IUE was performed at E14.5 to deliver shRNA\#1 or shRNA\#2 to neural progenitors, and the distribution of SON knockdown cells in the developing cortex at E18.5 was examined. As shown in Fig. 2c, GFP-positive Son knockdown neurons (shRNA\#1 and shRNA\#2) in the upper cortical plate (UCP) were sparse compared with GFPpositive neurons without Son knockdown (control). The quantification of GFP-positive cells in each cortical layer revealed that fewer Son knockdown neurons than control neurons were distributed in the UCP (Fig. 2d). Although slightly more Son knockdown cells than control cells were distributed in the lower cortical plate (LCP) and IZ, the differences were not statistically significant. In addition, we examined SON expression levels in GFPpositive shRNA-introduced cells in electroporated samples, and confirmed that the SON signals in GFP-positive cells was hardly detectable, while that in GFP-negative cells was clearly observed as nuclear speckles (Fig. 2e). These results indicate that canonical Son expression in neural progenitors is indispensable for normal neuronal migration.

Next, we performed rescue experiments by overexpressing shRNA-resistant human SON (hSONr) in knockdown cells. In addition, we examined constructs expressing two forms of disease-associated mutant SON. hSONm1 is a truncated mutant without most of the known functional domains, while hSONm2 lacks RNAbinding motifs and the C-terminal half of the RS domain (Fig. 3a). The former is derived from a SON mutation reported by Kim et al. [1], and the latter is from the most prevalent mutation found in ZTTK syndrome [1, 3, 4]. The effective production of hSONr, hSONm1, and hSONm2 in HEK293 cells in the presence of shRNA\#1 was confirmed (Fig. $3 \mathrm{~b}$ ). Then, the vectors expressing wild-type or mutant SON were introduced along with shRNA vectors into neural progenitors at E14.5, and the distribution of GFP-positive cells was examined. As shown in Fig. 3c and d, migration defects induced by shRNA\#1 were rescued by the overexpression of hSONr (shRNA\#1+hSONr), confirming that the defects were caused by SON insufficiency in neural progenitors. Intriguingly, the overexpression of hSONm2 (shRNA\#1+ hSONm2), but not that of hSONm1 (shRNA\#1+ hSONm1), rescued the defects as well, indicating that hSONm2, like hSONr, exerts sufficient functions for neuronal migration, while hSONm1 does not.

Since ID is not always accompanied by cerebral cortical malformation due to migration abnormalities, we reasoned that $S O N$ haploinsufficiency affects other factors essential for intellectual abilities. Therefore, we examined the dendritic spine density on Son knockdown neurons. The density of dendritic spines on Son knockdown cortical neurons at P60 $(7.6 \pm 0.5$ per $10 \mu \mathrm{m})$ was decreased by approximately $30 \%$ compared to that on control neurons $(10.6 \pm 0.9$ per $10 \mu \mathrm{m})$ (Fig. 4a, b). The forced expression of hSONr resulted in a dendritic spine density nearly equal to that on control neurons, confirming that $\mathrm{SON}$ insufficiency resulted in decreasing in the numbers of 

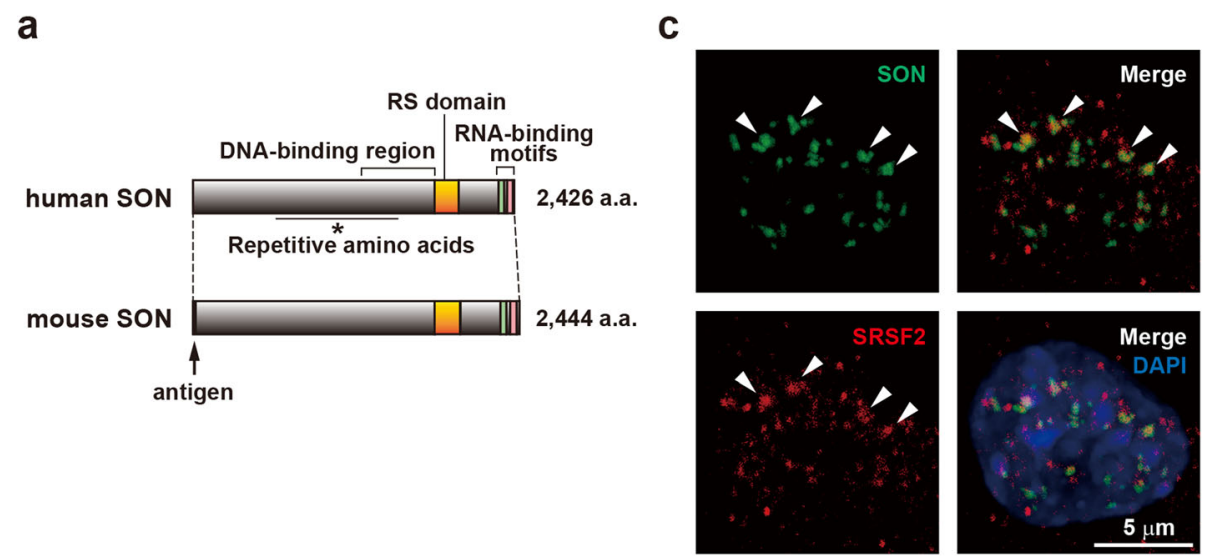

b

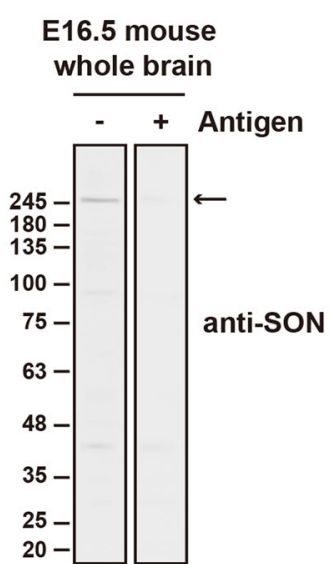

d

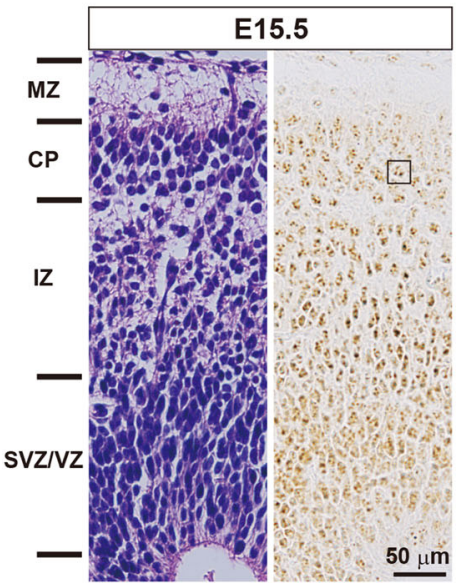

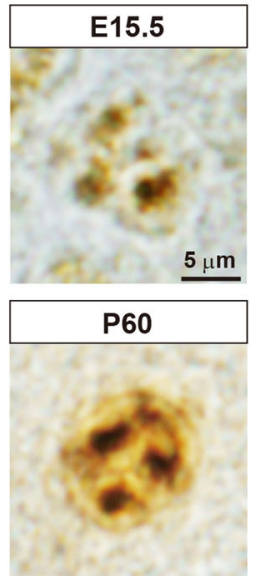

Fig. 1 SON distribution in neural cells and in the developing mouse brain cortex. (a) A schematic representation of the structure of full-length human and mouse SON. The arrow indicates the portion used as an antigen for antibody production. (b) Characterization of the anti-SON antibody. E16.5 mouse brain lysates were used for Western blotting. The antibody detected multiple bands. The major band, the 260-kDa band indicated by the arrow, was almost completely absent after the antibody was absorbed with glutathione s-transferase-fusion antigen peptide (right lane). (c) The localization of SON in the nucleus of Neuro-2a cells. Cells were stained with an anti-SON (green) or anti-SRSF2 (red) antibody. The nuclei were stained with DAPI (blue). The arrowheads indicate the colocalization of SON and SRSF2. (d) Immunohistochemical distribution of SON in the developing mouse brain and its subcellular localization in mature neurons. The left panel shows a hematoxylin and eosin (HE)-stained section of the E15.5 mouse cerebral cortex. The layered structure of the developing cortex is shown on the left. MZ: marginal zone; CP: cortical plate; IZ: intermediate zone; SVZ: subventricular zone; VZ: ventricular zone. The middle panel shows the distribution of SON in an adjacent section to the HE-stained section. At E15.5, most neuronal progenitors and neurons expressed SON. The right upper panel shows a higher magnification view of the boxed area in the middle panel. SON was localized to the nucleus and was present as speckles, as in cultured cells. A similar subcellular distribution of SON was observed in mature neurons at P60 (the left lower panel)

dendritic spines (Fig. 4a, b). These data indicate that SON is necessary for normal spine formation during neural development and that SON haploinsufficiency may cause dendritic spine abnormalities. Again, the overexpression of hSONm2, but not that of hSONm1, rescued the abnormalities as well, indicating that hSONm2 retains functions necessary for spine formation, while hSONm1 does not (Fig. 4a, b).

\section{Discussion}

In this report, we clarified that the canonical expression of Son is necessary for normal neuronal migration and dendritic spine formation in the developing mouse cerebral cortex. In addition, a truncated form of SON encoded by the most prevalent mutant SON identified in ZTTK syndrome patients can ameliorate the neuronal abnormalities induced by Son knockdown. These findings provided some hints to understand the pathophysiological mechanisms underlying the neural symptoms of ZTTK syndrome.

Among the 31 de novo mutations in the SON gene reported to be associated with ZTTK syndrome so far, twenty-eight encode truncated SON proteins due to either frameshift or nonsense substitutions that generate premature termination codons (Table 1). These truncated SON 
a

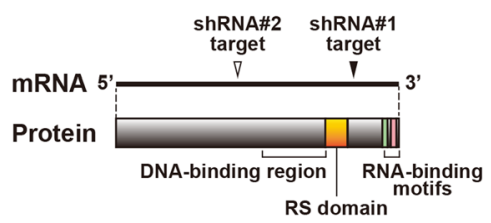

C

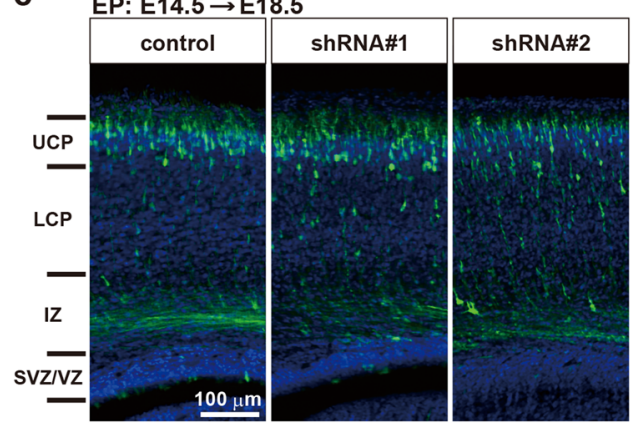

b

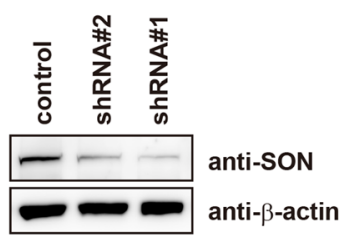

d

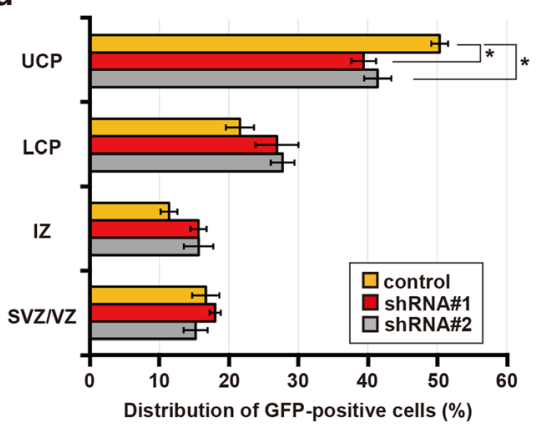

e

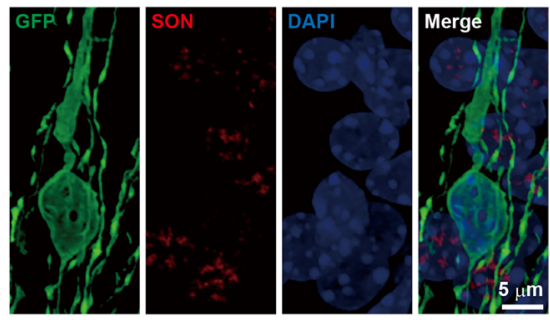

Fig. 2 SON is necessary for normal neuronal migration. a A schematic representation of the shRNA target sites. The closed and open arrowheads indicate the target positions of shRNA\#1 and shRNA\#2, respectively, on SON mRNA. $\mathbf{b}$ Characterization of Son shRNA-expressing vectors. Neuro-2a cells were transfected with parental pLLC vector (control) or vectors engineered to express Son shRNAs (shRNA\#1 or shRNA\#2). Cell lysates were subjected to Western blotting with an anti-SON antibody to assess the effect of knockdown. $\beta$-actin was used as a loading control and was used to normalize quantified values of SON signals. c Representative images showing the distribution of GFP-positive cells transfected with either empty vectors (control) or a vector expressing shRNA\#1 or shRNA\#2 in the developing mouse cerebral cortex. The layered structure is shown on the left. UCP: upper cortical plate; LCP: lower cortical plate; other abbreviations as described in Fig. 1d. The cortical plate is divided into the UCP and LCP according to cell density [18]. Transfection was performed by IUE at E14.5. Coronal brain sections were prepared at E18.5 and stained for GFP (green) and DAPI (blue). d The quantification of GFP-positive cells in each layer of the developing cortex. Each layer is described as in (c). Total numbers of GFP-positive cells studied in each brain ranged 306-567. Error bars represent standard error of the mean (SEM). $n=4$; ${ }^{*} p<0.05$ by one-way ANOVA followed by Dunnett's test. The original data are available in Additional file 1 [Table S1]. e The confirmation of Son knockdown in shRNA-introduced neurons. Coronal sections prepared as described in (c) were stained for GFP (green), SON (red), and DAPI (blue)

proteins, if produced, vary in length, with their C-termini distributed widely over the normal full-length SON protein and function differently from one another. However, mRNAs bearing a premature termination codon are often targeted by NMD, and are degraded [19]. Kim et al. revealed that some mutant $S O N$ mRNAs and proteins are indeed highly downregulated in the peripheral blood mononuclear cells of patients [1], suggesting that ZTTK syndrome is caused by SON haploinsufficiency; truncated proteins encoded by mutant SON are not involved in pathogenesis of ZTTK syndrome. The pathophysiological consequence of $S O N$ insufficiency in brain development is therefore of great interest to understand the neural symptoms of ZTTK syndrome. In this respect, our finding that the number of neurons that migrated into the UCP decreased by approximately $20 \%$ due to Son knockdown provides a concrete evidence that SON insufficiency in neural progenitors results in migration defects, which seems to be the pathological basis of brain malformation in ZTTK syndrome. More importantly, we found reduced spine density on Son knockdown neurons. Dendritic spine dysgenesis, such as a reduction in the number of spines and morphological abnormalities of spines, in cortical neurons was originally reported as a common pathological feature found in mentally retarded children with normal karyotypes [20]. The similar spine abnormalities of cortical neurons were identified also in genetic disorders associated with mental retardation, a former name for ID (reviewed 


\section{a}

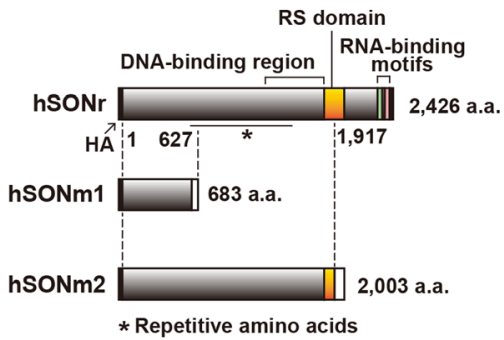

C
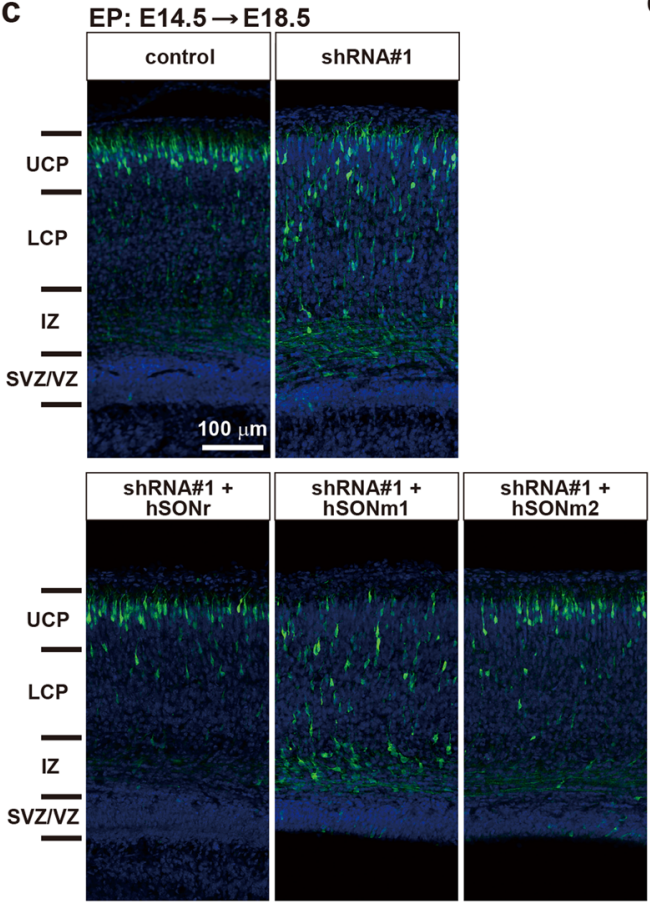

b

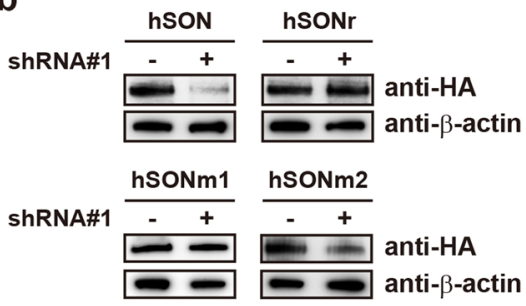

d

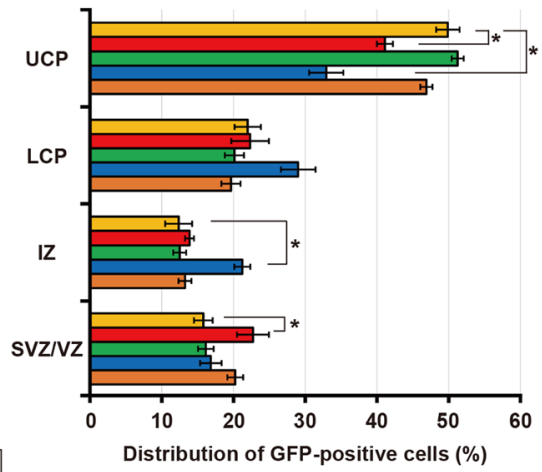

Fig. 3 Wild-type human SON and a disease-associated mutant SON rescue migration defects induced by Son knockdown. a Schematic representations of the structures of shRNA-resistant human SON (hSONr) and disease-associated mutant SON proteins (hSONm1 and hSONm2). (b) The confirmation of shRNA resistance of various forms of human SON. HEK293 cell lysates expressing various forms of hSON with or without shRNA\#1, as described above, were subjected to Western blotting with an anti-HA antibody. $\beta$-actin was used as a loading control. c Representative images showing the distribution of GFP-positive cells in the rescue experiments. The layered structure is shown as in Fig. 2c. Vectors expressing shRNA with or without those expressing various forms of hSON described in the boxes above each image were transfected into neural progenitors at E14.5. Parental pLLC vectors expressing GFP were used as a control. Coronal sections were prepared at E18.5 and stained as in Fig. 2c. d The quantification of GFP-positive cells in each layer of the developing cortex. Each layer is described as in (c). Total numbers of GFP-positive cells studied in each brain ranged $161-757$. Error bars represent the SEM. $n \geq 3 ;{ }^{*} p<0.05$ by one-way ANOVA followed by Dunnett's test. The original data are available in Additional file 1 [Table S2]

in [21]). Finally, the search for genetic causes of ID has identified numerous mutated genes that may be involved in synapse formation and the regulation of dendritic spine morphology [22]. To our knowledge, postmortem reports of ZTTK syndrome patients are unavailable. Therefore, reduced spine density on Son knockdown neurons is an important finding and suggests that spine defects are the pathological basis of ID in ZTTK syndrome.

Rescue experiments that involved the introduction of two forms of disease-associated mutant SON proteins confirmed that the truncated SON proteins encoded by mutant $S O N$ genes differ in their residual functions, even though both mutations cause ZTTK syndrome. As shown in Fig. 3a, hSONm1 is a severely truncated SON with 683 amino acids and hSONm2 is a mildly truncated SON with 2003 amino acids. Previous studies have revealed that the RS domain and the G-patch that constitutes RNA-binding motifs are the core motifs necessary for SON mediated RNA splicing [7] and a middle portion partially overlapping with a unique amino acid repeats is the DNA-binding region that bind to human genomic and viral DNAs $[9,23]$. Based on the domain structure, hSONm2 lacking an RNA-binding motif and part of the RS domain is expected to be functional in 
a

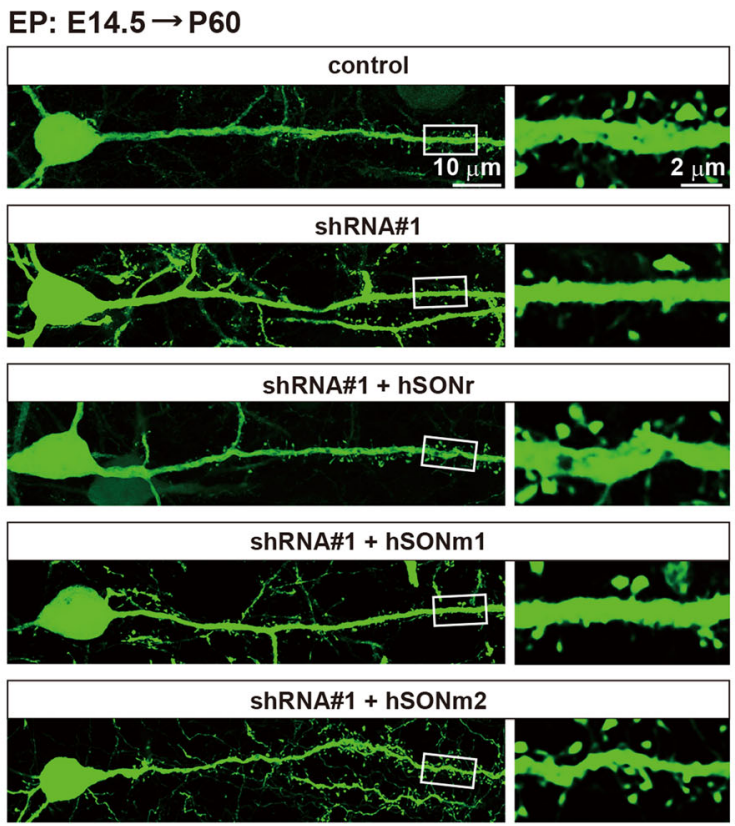

b

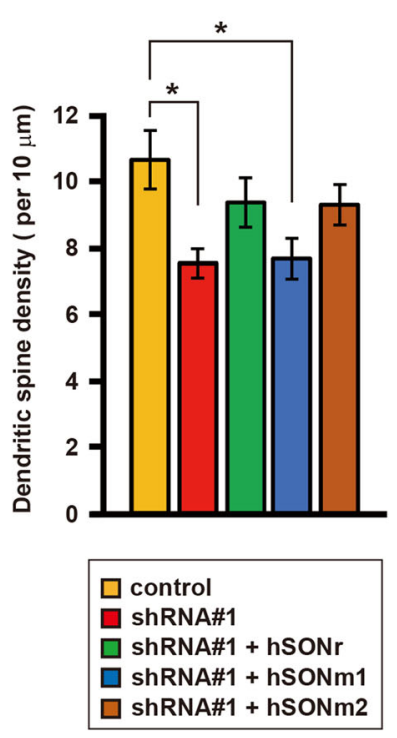

Fig. 4 Son knockdown causes a reduction in spine density on cortical pyramidal neurons. a Representative images showing pyramidal neurons in layers II/III at P60. Vectors expressing shRNA\#1 with or without those expressing wild-type (hSONr) or mutant hSON (hSONm1 or hSONm2), as described in the boxes above each image, were transfected into neural progenitors at E14.5. Parental pLLC vectors were used as controls. Coronal cortical sections were prepared at P60 and stained for GFP (green). The right panels are high magnification views of the boxed areas in the left panels. $\mathbf{b}$ Quantification of the spine density. The number of spines on each dendrite at between $30 \mu \mathrm{m}$ and $80 \mu \mathrm{m}$ from the soma was counted. The spine density is represented as the number of spines per dendrite length of $10 \mu \mathrm{m}$. Error bars represent the SEM. One-way ANOVA followed by Dunnett's test was used for each statistical analysis. ${ }^{*} p<0.05, n \geq 17$ neurons. The original data are available in Additional file 1 [Table S3]

part, as the DNA-binding domain remains intact. In contrast, hSONm1 lacking most of the identified domains were supposed to be hardly functioning. Indeed, the overexpression of hSONm2, like wild-type SON, successfully rescued the neuronal abnormalities, while the overexpression of hSONm1, failed to do so in our rescue experiments. These data indicate that the hSONm1-coding mutation is a loss-of-function mutation, but that the hSONm2-coding mutation retains function comparable, at least in the neural development, to that of wild-type SON. As mentioned above, it is suggested that ZTTK syndrome is caused by SON haploinsufficiency; potentially functional proteins encoded by mutant SONs are not involved in pathogenesis of ZTTK syndrome due to NMD [1]. It is, therefore, not surprising that hSONm2 encoded by the most prevalent mutant SON associated with ZTTK syndrome retained considerable function in neural development. Rather, the finding may further support noninvolvement of truncated proteins encoded by disease-associated mutant SONs in pathogenesis of ZTTK syndrome.

The molecular mechanisms underlying the neural abnormalities caused by SON mutations remain unclear because the roles of the multifunctional nuclear protein SON are diverse and not fully understood. The finding that hSONm2, which lacks an RNA-binding motif and part of the RS domain, behaved like wild-type SON in the rescue experiments may provide hints for these mechanisms. A simple explanation for this result is that the loss of the RNA splicing function of SON does not play a significant role in the observed neuronal abnormalities. Instead, other functions, such as transcriptional regulation, are more relevant to neural pathology since SON interacts with more than a thousand of genes via its DNA-binding region and is involved in the transcriptional repression of many target genes [9]. This is supported by the fact that rare non-truncating mutations, i.e., missense mutations [4] and an in-frame deletion [1], identified in ZTTK syndrome patients, are located exclusively in and around the genomic region that encodes the DNA-binding region (Table 1). However, there is a possibility that hSONm2 influences SON-mediated RNA splicing because of its structural similarity to SON E, a physiological isoform of SON. This truncated isoform, which lacks an RNA-binding motif, has been reported to enhance full-length SON-mediated RNA splicing [9]. Therefore, it is possible that hSONm2 together with residual mouse full-length SON rescues RNA splicing deficits caused by SON insufficiency. Many more investigations are necessary to understand the detailed molecular 
Table 1 Summary of mutation types identified in SON in association with ZTTK syndrome

\begin{tabular}{|c|c|c|c|c|}
\hline Mutation type & CDNA & Protein (predicted) & No. of case & Reference \\
\hline \multirow{13}{*}{$\begin{array}{l}\text { Frame-shift deletion } \\
\text { ( } 20 \text { cases) }\end{array}$} & c.268del & p.Ser90Valfs*59 & 1 & [1] \\
\hline & c.1881_1882del ${ }^{a}$ & p.Val629Alafs*56 & 1 & [1] \\
\hline & c.2365del & p.Ser789Alafs*8 & 1 & [1] \\
\hline & c.3597_3598del & p.Pro1200Argfs*17 & 1 & [1] \\
\hline & c.3852_3856del & p.Met1284llefs*2 & 2 & {$[1,4]$} \\
\hline & c.4055del & p.Pro1352Glnfs*14 & 1 & [1] \\
\hline & c.4358_4359del & p.Thr1453Serfs*11 & 1 & [1] \\
\hline & c.4640del & p.His1547Leufs*76 & 1 & [1] \\
\hline & c.4678del & p.Glu1560Lysfs*63 & 1 & [1] \\
\hline & c.5549_5550del & p.Arg1850llefs*3 & 1 & [1] \\
\hline & c.5753_5756del ${ }^{a}$ & p.Val1918Glufs*87 & 7 & {$[1,3,4]$} \\
\hline & c.6087del & p.Ser2029Argfs*22 & 1 & [1] \\
\hline & c.6233del & p.Pro $2078 \mathrm{Hisfs}^{*} 4$ & 1 & [4] \\
\hline \multirow[t]{2}{*}{$\begin{array}{l}\text { Frame-shift insertion } \\
(2 \text { cases) }\end{array}$} & c.[4999_5013del; 5031_5032insAA] ${ }^{\text {b }}$ & $\begin{array}{l}\text { p.[Asp1667_Asn1671del; } \\
\text { Asp1678Lysfs*9] }\end{array}$ & 1 & [1] \\
\hline & c.6002_6003insCC & p.Arg2002Glnfs*5 & 1 & [1] \\
\hline \multirow{2}{*}{$\begin{array}{l}\text { Frame-shift duplication } \\
\text { ( } 2 \text { cases) }\end{array}$} & c.3073dup & p.Met1025Asnfs*6 & 1 & [4] \\
\hline & c.4549dup & p.Glu1517Glyfs*6 & 1 & [1] \\
\hline \multirow{3}{*}{$\begin{array}{l}\text { Nonsense substitution } \\
\text { ( } 4 \text { cases) }\end{array}$} & c. $286 C>T$ & p.Gln $96^{*}$ & 1 & [4] \\
\hline & c.394C > T & p.Gln132* & 1 & [5] \\
\hline & c. $3334 C>T$ & p.Arg11112* & 2 & {$[1,2]$} \\
\hline In-frame deletion & c.4151_4174del24 & p.Leu1384_Val1391del & 1 & [1] \\
\hline Missense substitution & c. $[4909 \mathrm{~A}>\mathrm{T} ; 5528 \mathrm{C}>\mathrm{A}]^{\mathrm{C}}$ & p.[Thr1637Ser; Ser1848Thr] & 1 & [4] \\
\hline Whole gene deletion & - & - & 1 & [1] \\
\hline
\end{tabular}

${ }^{a}$ These two types of mutation were examined in this study. ${ }^{b}$ An in-frame deletion and a frame-shift insertion were identified in one allele; the latter was regarded as pathogenic. " Two substitutions were identified in one allele

mechanisms underlying the neural pathology of ZTTK syndrome, and accumulation of clinical and genetic information of ZTTK syndrome is also important.

In conclusion, this study revealed clearly that Son insufficiency results in neuronal migration defects and dendritic spine abnormalities in the mouse brain. Since information about the neuropathology of ZTTK syndrome is extremely limited, these findings provide important neuropathological basis possibly responsible for the neural symptoms, i.e., brain malformation and ID, of ZTTK syndrome. In addition, rescue experiments provided further evidence supporting that putative neural abnormalities in ZTTK syndrome are caused by $S O N$ haploinsufficiency regardless of the residual functions of mutant SON genes.

\section{Supplementary information}

Supplementary information accompanies this paper at https://doi.org/10. 1186/s13041-020-00622-4.

Additional file 1: Table S1. Numbers of GFP-positive cells in each layer of the developing cortex (\%) Table S2. Numbers of GFP-positive cells in each layer of the developing cortex (\%) Table S3. Dendritic spine density values (per $10 \mu \mathrm{m}$ of dendrite) of each neuron

\section{Abbreviations}

GFP: Green fluorescent protein; HA: Hemagglutinin; HE: Hematoxylin and eosin; HRP: Horseradish peroxidase; ID: Intellectual disability; IUE: In utero electroporation; IZ: Intermediate zone; LCP: Lower cortical plate; MZ: Marginal zone; NMD: Nonsense-mediated mRNA decay; PBS: Phosphate-buffered saline; SEM: Standard error of the mean; shRNA: Small hairpin RNA; SVZ: Subventricular zone; UCP: Upper cortical plate; VZ: Ventricular zone; ZTTK: Zhu-Tokita-Takenouchi-Kim

\section{Acknowledgments}

We thank Dr. Eun-Young Erin Ahn (Mitchell Cancer Institute University of South Alabama, USA) for SON constructs and a fruitful discussion, Dr. J. Miyazaki (Osaka University, Japan) for the pCAGGS vector, and Mr. R. Iwaki (Clinical Laboratory of Central Hospital, Aichi Developmental Disability Center) for preparing paraffin sections.

\section{Authors' contributions}

TM and AN contributed to the conception and design of the study. MU, MF, AT, Al, TM, SE and HT contributed to the acquisition and analysis of data. $\mathrm{MU}, \mathrm{TM}, \mathrm{MF}$, and AN contributed to drafting the manuscript and preparing the figures. AN contributed to preparing the table. All authors read and approved the final manuscript. 


\section{Funding}

This study was supported in part by Japan Society for the Promotion of Science (JSPS) KAKENHI grant numbers JP17K16292 to M.U., JP16K10202 to A.N., and JP15K06795 to T.M.

\section{Availability of data and materials}

All original data to generate Figs. 2, 3 and $4 d$ and $b$ are included in this published article [Table S1, S2, and S3 in Additional file 1 "Supplemental Tables"]. Materials are available on request.

\section{Ethics approval and consent to participate}

The animal experiments described in this manuscript were approved by the Animal Care and Use Committee of the Institute for Developmental Research.

\section{Consent for publication}

Not applicable.

\section{Competing interests}

The authors declare that they have no competing interests.

\section{Author details}

'Department of Cellular Pathology, Institute for Developmental Research, Aichi Developmental Disability Center, Kasugai, Aichi 4800392, Japan. 2 Department of Neurobiochemistry, Nagoya University School of Medicine, Nagoya, Aichi 4668560, Japan. ${ }^{3}$ Biogate Co. Ltd., 331-1 Ohmori, Yamagata, Gifu 5012123, Japan. ${ }^{4}$ Department of Molecular Neurobiology, Institute for Developmental Research, Aichi Developmental Disability Center, Kasugai, Aichi 4800392, Japan.

Received: 13 March 2020 Accepted: 12 May 2020

Published online: 24 May 2020

\section{References}

1. Kim JH, Shinde DN, Reijnders MRF, et al. De novo mutations in SON disrupt RNA splicing of genes essential for brain development and metabolism, causing an intellectual-disability syndrome. Am J Hum Genet. 2016;99(3): 711-9.

2. Kim J, Park EY, Chitayat D, et al. SON haploinsufficiency causes impaired premRNA splicing of CAKUT genes and heterogeneous renal phenotypes. Kidney Int. 2019;95(6):1494-504

3. Takenouchi T, Miura K, Uehara T, et al. Establishing SON in 21q22.11 as a cause a new syndromic form of intellectual disability: possible contribution to Braddock-Carey syndrome phenotype. Am J Med Genet Part A. 2016; 170(10):2587-90.

4. Tokita MJ, Braxton AA, Shao Y, et al. De novo truncating variants in SON cause intellectual disability, congenital malformations, and failure to thrive. Am J Hum Genet. 2016:99(3):720-7.

5. Yang $Y, X u L, Y u Z$, et al. Clinical and genetic analysis of ZTTK syndrome caused by SON heterozygous mutation C.394C>T. Mol Genet Genomic Med. 2019. https://doi.org/10.1002/mgg3.953.

6. Zhu X, Petrovski $S$, Xie $P$, et al. Whole-exome sequencing in undiagnosed genetic diseases: interpreting 119 trios. Genet Med. 2015;17(10):774-81.

7. Ahn EY, DeKelver RC, Lo MC, et al. SON controls cell-cycle progression by coordinated regulation of RNA splicing. Mol Cell. 2011;42(2):185-98.

8. Ahn EEY, Higashi T, Yan M, et al. SON protein regulates Gata-2 through transcriptional control of the microRNA 23a 27a 24-2 cluster. J Biol Chem. 2013;288(8):5381-8.

9. Kim JH, Baddoo MC, Park EY, et al. SON and its alternatively spliced isoforms control MLL complex-mediated H3K4me3 and transcription of leukemiaassociated genes. Mol Cell. 2016;61(6):859-73.

10. Lu X, Göke J, Sachs F, et al. SON connects the splicing-regulatory network with pluripotency in human embryonic stem cells. Nat Cell Biol. 2013;15(10): 1141-52.

11. Lu X, Ng HH, Bubulya PA. The role of SON in splicing, development and disease. Wiley Interdiscip Rev RNA. 2014;5(5):637-46.

12. Sharma A, Markey M, Torres-Muñoz $K$, et al. Son maintains accurate splicing for a subset of human pre-mRNAs. J Cell Sci. 2011;124(24):4286-98.

13. Wynn SL, Fisher RA, Pagel C, et al. Organization and conservation of the GART / SON / DONSON locus in mouse and human genomes mouse. Genomics. 2000;68(1):57-62.
14. Matsuki T, Matthews RT, Cooper JA, et al. Reelin and Stk25 have opposing roles in neuronal polarization and dendritic Golgi deployment. Cell. 2010; 143(5):826-36.

15. Sharma A, Takata $H$, Shibahara $K$, et al. Son is essential for nuclear speckle organization and cell cycle progression. Mo Biol Cell. 2010;21(4):650-63.

16. Tabata $\mathrm{H}$, Nakajima K. Efficient in utero gene transfer system to the developing mouse brain using electroporation: visualization of neuronal migration in the developing cortex. Neuroscience. 2001;103(4):865-72.

17. Fei J, Jadaliha M, Harmon TS, et al. Quantitative analysis of multilayer organization of proteins and RNA in nuclear speckles at super resolution. J Cell Sci. 2017;130(24):4180-92

18. Matsuki T, Chen J, Howell BW. Acute inactivation of the serine-threonine kinase Stk25 disrupts neuronal migration. Neural Dev. 2013;8(1):21.

19. Miller JN, Pearce DA. Nonsense-mediated decay in genetic disease: friend or foe? Mutat Res Rev Mutat Res. 2014;762:52-64.

20. Purpura DP. Dendritic spine "dysgenesis" and mental retardation. Science. 1974;186(4169):1126-8.

21. Kaufmann WE, Moser HW. Dendritic anomalies in disorders associated with mental retardation. Cereb Cortex. 2000;10:981-91.

22. Verpelli C, Sala C. Molecular and synaptic defects in intellectual disability syndromes. Curr Opin Neurobiol. 2012;22:530-6.

23. Sun $C T$, Lo $W Y$, Wang $\|_{\text {, }}$ et al. Transcriptional repression of human hepatitis $\mathrm{B}$ virus genes by negatively regulatory element-binding protein/SON. J Biol Chem. 2001;276:24059-67.

\section{Publisher's Note}

Springer Nature remains neutral with regard to jurisdictional claims in published maps and institutional affiliations.
Ready to submit your research? Choose BMC and benefit from:

- fast, convenient online submission

- thorough peer review by experienced researchers in your field

- rapid publication on acceptance

- support for research data, including large and complex data types

- gold Open Access which fosters wider collaboration and increased citations

- maximum visibility for your research: over $100 \mathrm{M}$ website views per year

At $\mathrm{BMC}$, research is always in progress.

Learn more biomedcentral.com/submissions 